Pierre-Alain Duc, Jonathan Braine and Elias Brinks, eds.

\title{
The Effect of Cosmic-Ray Diffusion for Parker Instability
}

\author{
Takuhito Kuwabara
}

Institute of Astronomy, National Central University, Chung-Li, Taiwan 320, R.O.C.

Kenji Nakamura

Matsue National College of Technology, Nishi-Ikoma-chou, Matsue-city, Shimane 690-8518, Japan

Chung-Ming Ko

Department of Physics, Institute of Astronomy and Center for Complex Systems, National Central University, Chung-Li, Taiwan 320, R.O.C.

\begin{abstract}
We studied the effect of anisotropic cosmic-ray (CR) diffusion for Parker instability by two-dimensional magnetohydrodynamic (MHD) simulation. We investigated two cases: a mechanical perturbation case and an explosional perturbation case. In the former case, the growth rate is proportional to the value of the diffusion coefficient of the CR. In the latter case, the growth rate becomes large in the early stages as the value of diffusion coefficient of the CR is getting small. But, after all, the result becomes the same as the former case in the late stages.
\end{abstract}

\section{Introduction}

The effect of $\mathrm{CR}$ is thought to have a large importance for the dynamics of the gas inside the galactic disk because the CR energy density is of the same order as that of the magnetic energy density and momentum energy of the gas. Recently, the linear analysis for the CR diffusion coefficient dependence of the Parker instability was studied by Ryu et al. (2003). And they showed that the growth rate is proportional to the value of the CR diffusion coefficient. On the other hand, the non-linear growth of the Parker instability with the effect of CR is not so clear even now, though there is a work by Hanasz \& Lesch (2000). In this study, we calculated the non-linear Parker instability with the effect of CR and checked how it grows under two kinds of perturbation.

\section{Results}

Figure 1 shows the schematic picture of the simulation region and the initial condition which is derived from the equilibrium balance under the constant gravity, plasma beta, alpha (the ratio of CR pressure to gas pressure), and the hot halo, the cold disk. The magnetic fields are horizontal initially. The units 


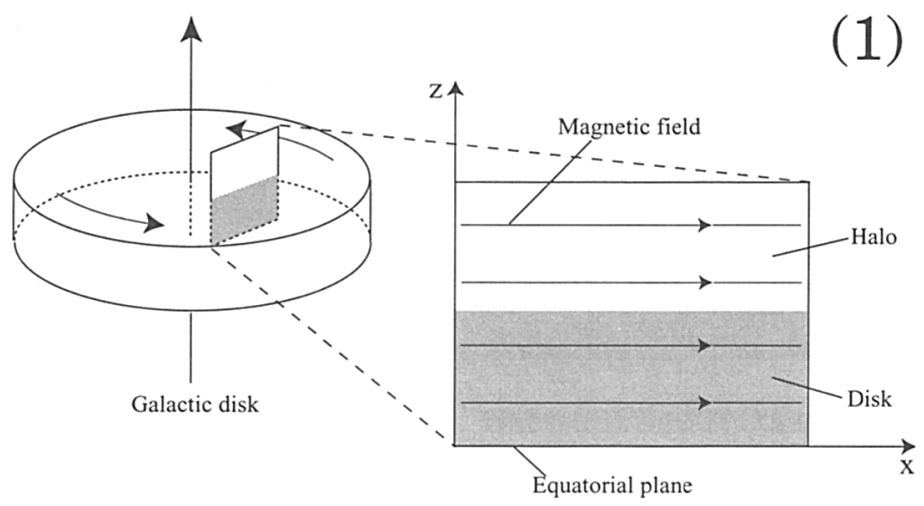

Figure 1. Schematic picture of the simulation region

are $\rho_{0}=1.6 \times 10^{-24} \mathrm{~g} \mathrm{~cm}^{-3}, H_{0}=50 \mathrm{pc}, C_{\mathrm{s} 0}=10 \mathrm{~km} \mathrm{~s}^{-1}$. The adiabatic indices are 1.05 for the gas and $4 / 3$ for the CR. The assumption that the CR energy diffuses only along the magnetic field lines is taken. We take the CR energy diffusion coefficient $\kappa_{\|}$as a parameter. Here, $\kappa_{\|}=3 \times 10^{28} \mathrm{~cm}^{2} \mathrm{~s}^{-1}$ equals 200 in our units.

Figure 2 shows the result in the case of the mechanical perturbation. Figure 2a, c shows the CR pressure distribution for $\kappa_{\|}=200$ and 10, a white line is a magnetic field line, and the arrows show velocity vectors, where a white arrow at the upper right corner shows a reference velocity vector $\left(=5 \times C_{\mathrm{s} 0}\right)$. The growth speed of large $\kappa_{\|}=200$ model is faster than the small $\kappa_{\|}=10$ model. Figure $2 \mathrm{~b}, \mathrm{~d}$ shows the $\mathrm{CR}$ pressure value along a magnetic field line displayed in Figure 2a, c. From these figures, we can recognize that the growth of the Parker instability is decelerated because the falling motion of the matter from the top to the foot point of the loop is prevented by the CR pressure gradient force in the small $\kappa_{\|}$model. Figure 3a, b shows the result in the case of the explosional perturbation for the large $\kappa_{\|}=100$ model and the small $\kappa_{\|}=10$ model. From this result, the growth speed of the Parker instability looks faster in small $\kappa_{\|}$ models than in large $\kappa_{\|}$models as shown by Hanasz \& Lesch (2000) contrary to the result in the case of a mechanical perturbation. But, the growth speed in large $\kappa_{\|}=100$ model becomes faster than small $\kappa_{\|}=10$ model later than the displayed time in this figure. In the early stages of the small $\kappa_{\|}$model, the $\mathrm{CR}$ remain close to the injection region for a long time and dominate the local dynamics. Subsequently, after the magnetic loop is beyond the disk surface, its growth is impeded by the CR pressure gradient force from the foot point to the top of the loop.

Acknowledgments. Numerical calculations were carried out on VPP5000 at NAOJ. TK and CMK are supported in part by the NSCT, NSC-91-2122-M008-006, NSC-91-2122-M-008-050. 

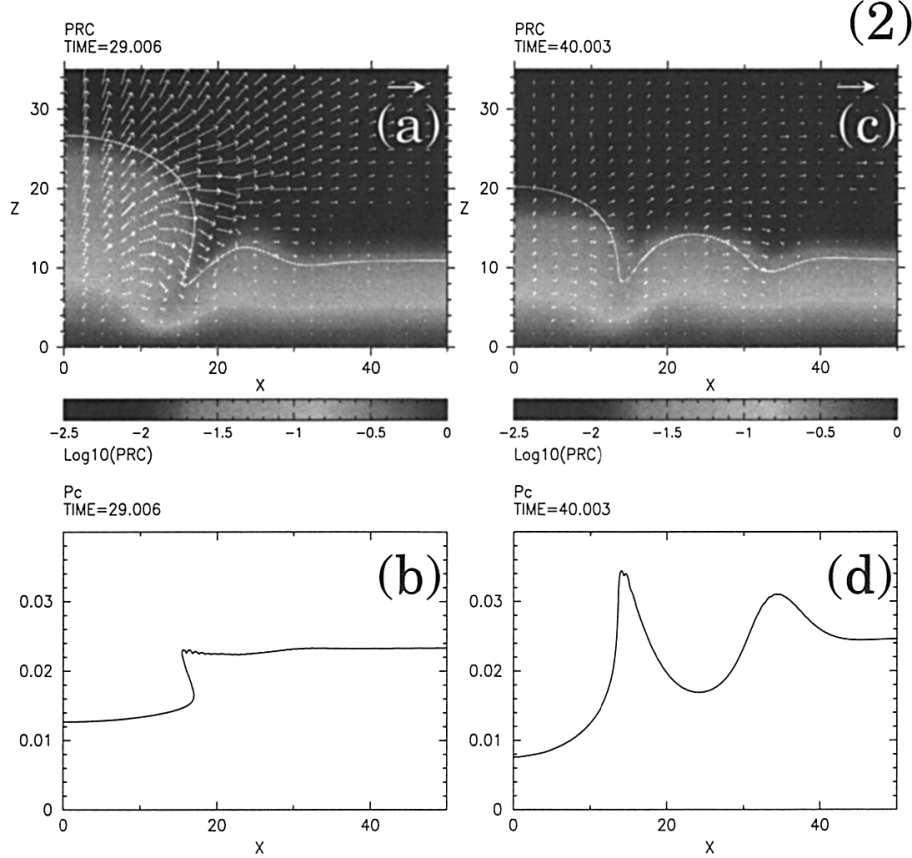

Figure 2. Result in the case of the mechanical perturbation
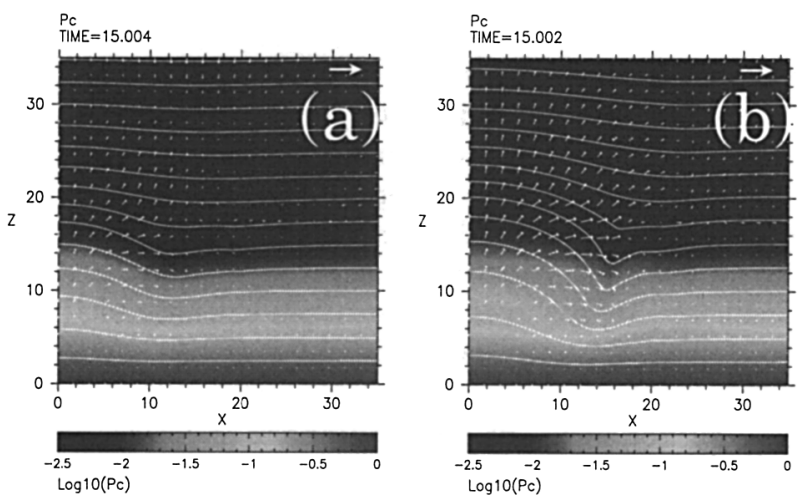

(3)

Figure 3. Result in the case of the explosional perturbation

\section{References}

Hanasz, M. \& Lesch, H. 2000, ApJ, 543, 235

Ryu, D., Kim, J., Hong, S. S. \& Jones, T. W. 2003, ApJ, 589, 338 